\title{
ACOLHIMENTO E PUERICULTURA: ESTRATÉGIAS DE HUMANIZAÇÃO DA ASSISTÊNCIA À CRIANÇA NA ATENÇÃO PRIMÁRIA À SAÚDE
}

\author{
RECEPTION AND CHILDCARE: STRATEGIES TO HUMANIZE CHILD \\ CARE IN PRIMARY HEALTH CARE
}

\author{
Gabrielle Sousa Amorim ${ }^{1}$ \\ Fernanda Jorge Magalhães ${ }^{2}$ \\ Karla Maria Carneiro Rolim ${ }^{3}$ \\ Miriam Calíope Dantas Pinheiro ${ }^{4}$ \\ Ana Valeska Siebra e Silva ${ }^{5}$ \\ Deyce Kelly Ponte Batista ${ }^{6}$
}

RESUMO: INTRODUÇÃO: O acolhimento à criança na atenção primária de saúde é uma ação que deve ocorrer em todos os locais e por todos os profissionais do serviço de saúde e visa promover vínculos entre os profissionais e usuários. Objetivo: Compreender a percepção de profissionais atuantes em uma unidade de atenção primária acerca do acolhimento à criança na consulta de puericultura. METODOS: Pesquisa descritiva, qualitativa, realizada em uma Unidade de Atenção Primária em saúde de um município do Maranhão-Brasil. Realizou-se uma entrevista semi-estruturada com 14 profissionais, analisando as falas por categorização. RESULTADOS: Foram evidenciadas informações por profissionais do serviço acerca do acolhimento como estratégia eficaz na humanização da assistência à criança na puericultura. CONCLUSÃO: Constatou-se a necessidade de capacitação

\footnotetext{
${ }^{1}$ Enfermeira.Especialista em Enfermagem Neonatal pelo Instituto e Saúde e Gestão Hospitalar, Brasil. gabrieleamorim23@yahoo.com.br.

${ }^{2}$ Doutora em Enfermagem pela Universidade Federal do Ceará (UFC). Doutora de Mobilidade Acadêmica. Internacional pela Universidade do Porto (UP), Portugal. Docente da Universidade de Pernambuco / Faculdade de Enfermagem Nossa Senhora das Graças (UPE/FENSG). fernandajmagalhaes@yahoo.com.br.

${ }^{3}$ Pós-doutora em Humanisationdes Soins em Néonatologie pela Universidade de Rouen (CHUROUEN), França. Coordenadora do Mestrado Profissional em Tecnologia e Inovação em Enfermagem da Universidade de Fortaleza (MPTIE/UNIFOR), Brasil. Docente Titular do Programa em Saúde Coletiva (PPGSC/UNIFOR). karlarolim@unifor.br.

${ }^{4}$ Doutora pela Universidade Federal do Rio de Janeiro (UFRJ), Brasil. Docente do MPTIE/UNIFOR. caliope@unifor.br.

${ }_{5}^{5}$ Doutora pela Universidade de São Paulo (USP), Brasil. Professorada Universidade Estadual do Ceará (UECE). anavaleska@usp.br.

${ }^{6}$ Enfermeira. Graduada pelo Centro Universitário Fametro - UNIFAMETRO. Kelly.deyce@gmail.com.
} 
de todos os profissionais envolvidos assim como dos gestores, de modo a sensibilizá-los para uma prática integral, com melhoria da assistência e favorecimento à promoção da saúde da criança.

Descritores: Puericultura; Conhecimento; Profissionais.

SUMMARY: INTRODUCTION: Child care in primary health care is an action that must take place in all places and by all professionals of the health service and aims to promote links between professionals and users, ensuring their rights, and their families. It aims to understand the perception of professionals working in a primary care unit about child care in the child care clinic. METHODOLOGY: Descriptive, qualitative research carried out in a Primary Health Care Unit of a municipality of Maranhão, Brazil. A semi-structured interview was conducted with 14 professionals, analyzing the statements by categorization and according to the literature. RESULTS: Information was provided by service professionals about the reception as an effective strategy in the humanization of child care in childcare. Some did not know what was child care and the difficulties of accepting consultations for the prevention of injuries, only depicting the illness. CONCLUSION: It was verified the need for training of all professionals involved as well as managers, in order to sensitize them to an integral practice, with improved care and favoring the promotion of children's health.

Keywords: Childhood; Knowledge; Professionals. 


\section{INTRODUÇÃO}

A Atenção Primária à Saúde é a porta de entrada das pessoas no Sistema Único de Saúde (SUS), assumindo o papel de oferecer cuidados primários de qualidade às crianças, tendo como foco a promoção da saúde e prevenção de agravos. Baseia-se em princípios como: longitudinalidade e integralidade da assistência, primeiro contato e coordenação do cuidado. Atua em uma área territorial definida e, dessa forma, tem condições de identificar e acompanhar as crianças sob sua responsabilidade, de modo a manter constante vigilância dos fatores determinantes e condicionantes da saúde ${ }^{(1)}$.

A promoção do cuidado deve ser desenvolvida através do acolhimento das necessidades de cada família, juntamente com a interação dos profissionais de saúde, para a construção de um relacionamento harmonioso, usando-se das tecnologias simples e estimuladoras do aprendizado oportunizando maior resolubilidade em solucionar problemas individuais e coletivos demandados pela população(2).

A puericultura é um dos programas do Ministério da Saúde (MS) utilizados no acompanhamento sistemático do crescimento e desenvolvimento de crianças 0 a 5 anos de idades, nas Unidades Básicas de Saúde (UBS) assim como na Estratégia Saúde da Família (ESF), bem como promover e manter a saúde, reduzir incidências de doenças e aumentar as chances desta alcançar todo o seu potencial ${ }^{(3)}$.

O aumento da cobertura da ESF está ligado à redução da mortalidade infantil e à maior promoção da equidade. Além disso, também trazem como resultados a maior qualidade da atenção pré-natal, da puericultura e da oferta de utilização dos serviços de saúde, apesar da baixa efetividade em todo sistema público de saúde ${ }^{(4)}$.

Para garantir a qualidade do acompanhamento à criança, o MS recomenda sete consultas bimestrais no primeiro ano de vida, duas consultas semestrais no segundo e, a partir desta idade, consultas anuais. Tais consultas resultam num conjunto de medidas de cuidados preventivos, que observam a criança dentro do 
seu contexto de vida e adequam-se à sua realidade, buscando compreender necessidades individuais e estabelecer vínculos entre a família, o serviço e a equipe de saúde ${ }^{(2)}$.

O calendário mínimo de consultas de puericultura, muitas vezes, não está sendo priorizado pelas mães, talvez se houvessem mais informações sobre a importância da puericultura e do acolhimento, bem como uma escuta qualificada à essas mães, evitaria consultas de intercorrências, que geralmente são fundadas em algum problema de saúde apresentado pela criança. Tais consultas são voltadas ao cumprimento de práticas majoritariamente curativas, e evidencia o desconhecimento da importância do acompanhamento, que leva à promoção da saúde ${ }^{(2)}$.

As gestantes devem ser sensibilizadas ainda no pré-natal para a relevância do acompanhamento regular da criança. Diante das considerações objetivou-se com o estudo compreender a percepção de profissionais atuantes em uma Unidade de Atenção Primária acerca da importância do acolhimento à criança na consulta de puericultura.

\section{MÉTODO}

Trata-se de pesquisa do tipo descritiva, com abordagem qualitativa. Descritiva porque tem como objetivo descrever as opiniões, atitudes e crenças de uma determinada população, além de proporcionar uma nova visão sobre a atuação prática do assunto abordado ${ }^{(6)}$.

Tem abordagem qualitativa por permitir representar a busca de conhecimento nas relações entre os seres humanos pela interpretação da realidade vivenciada através de falas, com o intuito de um melhor entendimento entre a prática e o conhecimento diante de uma situação ${ }^{(7)}$.

A pesquisa foi realizada em dezembro de 2016, em uma Unidade Básica de Saúde do município de Caxias - Maranhão, Brasil, que acolhe e promove o atendimento de saúde de duas equipes de saúde da família e que atende uma grande demanda da população. 
A demanda da comunidade para consulta com a enfermeira da UBS é, em média, de 20 pessoas por turno. A procura pelo médico é mais limitada devido à quantidade de atendimentos, com média de 15 consultas no dia em que este profissional se encontra na Unidade. A procura pelo profissional dentista é mais restrita ainda, na maioria dos casos devido à deficiência de algum equipamento, o que acaba interferindo na demanda deste serviço. A rotatividade dos usuários pelos serviços ofertados é grande, haja vista que a UBS possui uma sala de vacina e de procedimentos, a qual é utilizada para aplicação de medicamentos, realização de curativos, entrega de materiais para exames e também recebimento dos exames realizados.

Em relação ao número de consultas de enfermagem e/ou médica voltadas para a criança, fica em torno de 40 consultas/mês. No entanto, todas as consultas ocorrem devido a alguma queixa ou sintoma que o infante manifesta naquele momento, não havendo consulta programada para o acompanhamento do desenvolvimento e crescimento do mesmo.

Na consulta voltada para a criança faz-se necessário que o profissional cultive a sensibilidade, promovendo o cuidado por meio de comunicação efetiva e da atenção individualizada, reconhecendo às necessidades vigentes de cada mãe, respondendo aos apelos surgidos ao desenvolver o cuidado à criança. Acolher bem toda a família, para que adquiram confiança em si e nos profissionais e, consequentemente retornem ao serviço para o acompanhamento do crescimento e desenvolvimento da criança ${ }^{(2)}$.

Percebe-se a adesão das gestantes no acompanhamento pré-natal, contudo não é frequente a busca pela consulta de puericultura para acompanhamento do crescimento e desenvolvimento da criança, mas sim, para o tratamento do problema de saúde que acomete o infante no momento da procura. Esse fato pode ser comprovado pelos registros de atendimento da UBS, como também pelo prontuário da criança.

Os participantes de estudo foram 14 profissionais da equipe de saúde que trabalhavam na Unidade, os quais estavam em período regular das atividades, sendo estes uma enfermeira, um médico, um dentista, um técnico de enfermagem, um auxiliar administrativo, um vigia e oito agentes comunitários de saúde. Foram 
excluídos aqueles profissionais que estavam afastados de suas atividades, mesmo que temporariamente, fosse por gozo de férias ou licença. Os participantes foram entrevistados em local privativo e confortável, utilizando- se gravador para registro das falas, que foram posteriormente transcritas na íntegra. Os dados foram analisados à luz da literatura pertinente, bem como análise de conteúdo, gerando categorias, conforme associação das falas ${ }^{(8)}$.

Para organização dos dados e identificação das falas, foram utilizados letras e números para caracterizar a fala de cada participante. A participação no estudo foi voluntária, mediante leitura e assinatura de Termo de Consentimento Livre e Esclarecido (9). O estudo foi aprovado pelo Comitê de Ética e Pesquisa do Instituto de Saúde e Gestão Hospitalar, conforme Parecer n. 1.882.326.

\section{RESULTADOS}

Diante das falas dos participantes, foi possível identificar palavras correlatas com sentidos associados, o que gerou três principais categorias: "Conhecendo a puericultura"; "Fatores intervenientes para a puericultura" e "Acolhimento pela equipe de saúde".

\section{Conhecendo a puericultura}

Ao questionar os participantes sobre puericultura, foi perceptível o conhecimento ora consistente, ora superficial sobre o programa:

É uma consulta para o acompanhamento da criança, no sentido de estar observando o peso, o crescimento dela, e a partir daí é traçar algumas metas que pode dizer o que 
está bom, o que não está e o que pode ser melhorado (E4).

Puericultura é aquela parte da saúde destinada à atenção, digamos assim, à mãe e à criança recém-nascida (E8).

É o atendimento exclusivo à criança desde os primeiros dias de vida até a vida adulta (E 11).

Percebe-se nas falas a carência de padronização quanto à definição sobre o programa de puericultura perante o conhecimento da equipe de saúde, embora oito dos participantes tenham compreensão limitada de que este seja um atendimento voltado para o acompanhamento preventivo de saúde da criança.

A importância da puericultura está nos cuidados a criança [...] assim, eu acredito que seja. Principalmente, com a mãe de primeira viagem, que não sabe como cuidar e nem como se cuidar, sendo importante para orientar as mães de como se cuidar e de como cuidar do recémnascido (E 5).

Para nós, nem existia puericultura porque nós trabalhávamos com as crianças. Trabalhava com as crianças só para orientar na vacinação (E 2).

O que eu sei é que uma criança quando nasce, depois que chega em casa, a mãe precisa trazer para fazer avaliação... ver como está o bebê, se está mamando direitinho. Eu acho que é isso (E 7).

Durante as entrevistas, dois funcionários não souberam responder o que era puericultura, e nem dizer qual seria sua importância. Quando indagados sobre que 
profissional poderia realizar a puericultura, um deles respondeu que somente o médico poderia, e três afirmaram que seria a enfermeira, mas expressando incerteza. Além do superficial conhecimento pelos profissionais, outros fatores podem interferir de forma negativa na adesão a puericultura.

\section{Fatores intervenientes na puericultura}

As falas demonstraram fatores intervenientes na realização da puericultura, entre eles a busca pelo atendimento em saúde somente em situações de urgência:

Ela só vem quando ele está doente. Urgência todo mundo sabe que é no hospital e aqui é consulta mensal. Quando me falava que era urgência, eu mandava marcar consulta e, quando marcavam de hoje para amanhã, elas diziam que não vinham porque demorava demais, que chegava aqui e não dava certo. $E$ foi daí que começou a inventar a puericultura (E 2).

Tem mãe que não quer vir ao posto, não quer chegar e esperar para ser atendida. Se ela chegar e for a primeira a ser atendida tudo bem, se não, ela diz que vai passar o dia esperando que não precisa de nada porque o bebê não está doendo (E 7).

A dificuldade de comparecimento da criança é o principal fator. Talvez pela falta de conhecimento, da baixa renda (E 1).

Um dos fatores é o esclarecimento, para essa mãezinha, para essa família da importância de está trazendo a 
criança desde cedo e não trazer apenas quando está doente, mas prevenir que essa criança fique doente (E 6).

Sensibilizar o agente comunitário de saúde sobre a necessidade da consulta de puericultura (E 12).

Outro fator interveniente percebido foi a necessidade do esclarecimento às mães ou responsáveis sobre a consulta de puericultura, enfatizada pela maioria dos entrevistados como sendo uma alternativa que poderia contribuir para uma melhor adesão da família e da criança. A forma como a equipe de saúde recebe essa família também contribui para essa adesão.

\section{O acolhimento pela equipe de saúde}

A forma como o usuário é acolhido pela equipe e funcionários da UBS repercute na adesão aos programas trabalhados e desenvolvidos pela equipe, fato que também foi mencionado por parte dos entrevistados como queixas do usuário.

Não tendo um bom acolhimento, é besteira, mas tanto pela visitação do agente como vim ao posto, pelo modo de ser recebido até mesmo na maternidade, tudo isso dificulta trazer a mulher no pós-parto (E 5).

O acolhimento na unidade de saúde, é como se diz, a dificuldade na marcação (E 10).

O descomprometimento dos profissionais, estar acompanhando essa mãe, o acolhimento do serviço, fazendo com que essas se sintam prejudicadas e não compareçam mais (E 11). 
É importante desenvolver trabalhos com todos os integrantes da equipe, não somente aqueles relacionados aos programas de saúde. Além disso, buscar sensibilizar a todos quanto à humanização no atendimento ao usuário que busca o serviço, e entre os próprios funcionários, para que haja maior adesão das famílias no acompanhamento do crescimento e desenvolvimento da criança.

\section{DISCUSSÃO}

A Atenção Primária precisa ser avaliada continuamente, já que é notável sua colaboração no processo de melhorias do SUS. Não deve ser encarada como um elemento punitivo, mas um meio para implementar reformas nos sistemas de saúde com o objetivo de obter melhores resultados ${ }^{(10)}$.

Parte da equipe demonstrou conhecer o objetivo principal da consulta de puericultura. Alguns demonstraram déficit no conhecimento e confusão quanto à proposta de assistência em saúde durante a puericultura, conforme foi possível avaliar nas falas dos entrevistados sobre o conceito e objetivo da puericultura.

Percebeu-se, ainda, desconhecimento por parte dos funcionários, mesmo daqueles que não atuavam diretamente na assistência, mas que estavam exercendo uma atividade em uma unidade de saúde, pois é importante que conheçam algumas rotinas e programas relativos ao funcionamento da UBS.

A consulta de puericultura ainda é confundida não somente pela população, mas, especialmente, pelos agentes comunitários de saúde, que são elos da UBS com a comunidade. Estes a entendem como uma consulta de finalidade curativa, em que a comunidade, orientada de forma errônea, somente busca o atendimento para a criança em caso de urgência.

As consultas estão ocorrendo conforme o adoecimento, não se trabalhando a prevenção. Foi constatado que o pensamento voltado para a lógica da promoção e prevenção da saúde ainda não está presente na população, que busca soluções apenas para doenças já instaladas ${ }^{(4)}$. 
Muitas crianças frequentam periodicamente a UBS para consulta médica quando estão doentes, mas não por prevenção. A falta de orientação e comunicação adequadas motivam as mães a não levarem seus filhos às consultas de puericultura, pois consideram tal atendimento "sem seriedade", já que o filho não está doente, o que demonstra a falta de conhecimento a respeito da finalidade e importância com relação à atenção à saúde da criança ${ }^{11)}$.

A saúde da criança é o compromisso dos profissionais de saúde. Portanto, conhecer e orientar os pais sobre os cuidados com a saúde da criança deve ser prioridade nos atendimentos de saúde prestados à essa população. Essas informações facilitam o diálogo e o aconselhamento da mãe ou responsável, tornando a família aliada do serviço de saúde ${ }^{(12)}$.

É possível entender que se o profissional de saúde não tem o conhecimento necessário sobre o programa e consciência de sua relevância, de fato não conseguirá sensibilizar a população para que traga a criança que está sadia para um acompanhamento de forma regular, fato que pode explicar a não adesão da comunidade às consultas de puericultura. Ademais, o conhecimento limitado sobre $o$ programa ou a falta total deste conhecimento, a exemplo dos entrevistados AD1 e V1, que não responderam às perguntas sobre o conceito e o objetivo.

As morbidades e hospitalizações por causas evitáveis em crianças menores de quatro anos que ocorrem no país, como afecções respiratórias, doenças infecciosas ou parasitárias, doenças do aparelho digestivo, por causas externas e, as doenças do aparelho geniturinário poderiam ser evitadas a partir de um conjunto de ações sensíveis e resolutivas ainda na atenção primária, mas para que isto aconteça, faz-se necessário que se difunda a importância do seguimento periódico e contínuo dos programas de puericultura ${ }^{(13)}$.

Entre outras causas para a baixa adesão das famílias, além da falta de informações ou informações incompletas por parte da equipe de saúde, a ausência da equipe durante as consultas, contribui para a desinformação acerca da importância da puericultura ${ }^{(14)}$.

Percebe-se que existe um vínculo entre a população e os profissionais que ali atuam, atributo importante e desejado para que possa ocorrer uma cooperação mútua, em que o serviço seja uma fonte reguladora de atenção e que a população 
possa ter esse serviço como referência por um período de tempo. Essa relação entre o profissional e a população proporciona maior conhecimento dos problemas, com maior aproximação e confiança entre as partes e, consequentemente, maior potencial de resolução de tais problemas e emponderamento do indivíduo, como também das famílias destas crianças ${ }^{(10)}$.

Em toda equipe de saúde da Atenção Primária, a maior parte dos profissionais é agente comunitário de saúde, sendo a ponte de ligação entre o posto e a comunidade, e seu empenho e produção reflete no cotidiano da unidade de saúde e na situação de saúde da população da sua micro área.

É fundamental que o agente comunitário de saúde seja capacitado, estabelecendo relações que favoreçam à comunidade. Isso se dá por meio de investimentos em programas de educação em saúde, resultando não apenas nas mudanças de indicadores epidemiológicos, mas em mudanças de hábitos e concepções inadequadas ${ }^{(15)}$.

É preciso investir na qualificação da formação das equipes de Saúde da Família e da Atenção Primária, com a inserção de todos os profissionais no cuidado com a criança, tendo em vista a promoção e prevenção em saúde e, por conseguinte, a melhor qualidade de vida dessa população(13).

A maioria dos entrevistados citou que o bom acolhimento aos usuários da UBS é essencial para que ocorra adesão aos serviços oferecidos pela instituição, afinal, o comum é retornar onde se é bem recebido.

O acolhimento é a abertura dos serviços para a demanda, buscando garantir a satisfação do usuário. Desta maneira, modifica-se o processo de trabalho com intuito de garantir acesso e resolutividade e/ou referenciar, se necessário $\left({ }^{5)}\right.$.

O vínculo e a humanização entre os funcionários, comunidade e usuário da Atenção Primária, devem ser trabalhados, a fim de que a rotina do serviço de saúde seja desenvolvida com bom acolhimento.

Para fortalecer o vínculo com a mãe e a família faz-se necessário sensibilizálas acerca da importância das consultas de puericultura, investindo em ações de promoção da saúde, orientações diversas quanto aos cuidados gerais, incentivando o empoderamento dos sujeitos envolvidos e consequentemente fortalecendo o 
vínculo entre profissionais de saúde e as famílias, percebendo-se uma maior aceitação dos aprendizados proporcionados durante as consultas ${ }^{(2)}$.

É importante a equipe valorizar o momento do atendimento para que os responsáveis percebam a importância da puericultura, fazendo com que o usuário se sinta valorizado, seguro e esclarecido, facilitando o retorno deste nas consultas subsequentes, compreendendo os reais benefícios do acompanhamento ${ }^{(14)}$.

Os profissionais que atuam direta ou indiretamente na assistência à mãe e seu filho poderão contribuir para a promoção da saúde da criança, bem como oferecer contribuições significativas no estabelecimento de vínculo da mãe com a UBS, garantindo maior adesão à consulta de puericultura ${ }^{(11)}$.

\section{CONCLUSÃO}

Percebeu-se as disparidades quanto à definição sobre o programa de puericultura perante o conhecimento da equipe de saúde, o que torna imprescindível desenvolver atividades de capacitação entre os profissionais de saúde, para que toda a equipe tenha conhecimento acerca do papel de cada um dentro dos programas desenvolvidos nas UBS e, principalmente, para que todos conheçam a relevância de cada programa trabalhado, que no caso deste estudo foi sobre a puericultura.

Os profissionais de saúde, especialmente o agente comunitário de saúde e também os funcionários do posto (mesmo estes não sendo profissionais de saúde), devem ter conhecimento sobre a puericultura, já que estes, além de fazer parte da comunidade, estão sempre em contato com todos os usuários. Portanto, devem acolhê-los de forma humanizada e direcioná-los corretamente para a assistência a ser prestada.

O fato das entrevistas terem ocorrido durante o horário de trabalho dos funcionários pode ter contribuído para que alguns não aceitassem participar da pesquisa, bem como o período de transição da prefeitura do município, devido ao medo de perder o trabalho, por acreditarem que a pesquisa tivesse alguma relação 
com a futura gestão; o que certamente não permitiu melhor retrato do conhecimento da equipe sobre puericultura.

Assim, faz-se necessárias intervenções para que se busque conhecer a relevância da puericultura entre todos os profissionais de saúde, pois, dessa forma, será possível contribuir para a sensibilização de toda a comunidade sobre a importância de se levar as crianças para as consultas de acompanhamento do crescimento e desenvolvimento, ainda que estas não apresentem problemas de saúde, para que, de fato, se possa promover a saúde e prevenir doenças evitáveis. 


\section{REFERÊNCIAS BIBLIOGRÁFICAS}

ALMEIDA ER, MOUTINHO CB, CARVALHO SAS, et al. Report about the construction of a nursing protocol in child care in primary care. Rev Enferm UFPE on line., 2016; 10(2):68391.

SOARES DG, PINHEIRO MCX, QUEIROZ DM, SOARES DG. Implementation of childcare and care challenges in the Family Health Strategy in a city of the Ceará state. Rev Bras Promoç Saúde, 2016; 29(1): 132-8.

LIMA KF, FERNANDES NT. A relevância da puericultura na atenção primária a saúde. In: Anais... 17 SEMINÁRIO NACIONAL DE PESQUISA EM ENFERMAGEM. Rio Grande do Norte: ABEn-RN, 2013.2 Disponível em:<http://www.abeneventos.com.br/anais_senpe/17senpe/pdf/0160po.pdf>. Acesso em: 10 jul. 2017.

OLIVEIRA VBCA, VERISSIMO MLOR. Assistência à saúde da criança segundo suas famílias: comparação entre modelos de Atenção Primária. Rev Esc Enferm USP., 2015; 49(1): 30-6.

MARTINIANO CS, SOUZA FF. Análise dos cuidados de puericultura e da relação puericultor- criança cuidador na atenção básica. Rev. Bras. Pesq. Saúde, 2013; 15(1): 40-8.

GIL AC. Como elaborar projetos de pesquisa. 5ed São Paulo: Atlas, 2010. Gomes IM, et al. Use of external interviewers in qualitative research: action plan. Enfermería Global, 2016; 44(5): $245-54$.

BARDIN L. Análise de Conteúdo. São Paulo: Edições 70, 2011. Brasil. Ministério da Saúde. Conselho Nacional de Saúde. Comissão Nacional de Ética em Pesquisa - CONEP. Resolução $\mathrm{n}^{\circ}$ 466, de 12 de dezembro de 2012. Diretrizes e Normas Regulamentadoras de Pesquisa Envolvendo Seres Humanos. Brasília, DF, 2012.

BATISTA VCL, et al. Evaluation of primary health care attributes according to family health Professionals. SANARE, 2016; 15(2): 87-93.

MALAQUIAS TSM, Gaívab MAM, Higarashic IH. Perceptions of the family members of children regarding well-child check-ups in the family healthcare strategy. Rev Gaúcha Enferm., 2015;36(1):62-8.

CARVALHO EB, SARINHO SW. The nursing consultation in monitoring child growth and development in the family health strategy. Rev enferm UFPE on line., 2016;10(Supl. 6):480412.

ARAÚJO JP, SILVA RMM, Collet N. História da saúde da criança: conquistas, políticas e perspectivas. Rev Bras Enferm., 2014; 67(6): 1000-7.

GUIMARÃES TC. Puericultura: baixa adesão no PSF em Paracatu-Mg. Trabalho de Conclusão de Curso (Especialização em Estratégia Saúde da Família). Universidade Federal de Mato Grosso do Sul, Corumbá/MS, 2015.

PAULA RAO, et al. Community Health Agents' knowledge about Food Safety and an intervention. Rev. APS. 2015; 8(1): 16-21. 